\title{
RhoC/ROCK2 promotes vasculogenic mimicry formation through ERK/MMP and FAK/paxillin signaling pathways in hepatocellular carcinoma
}

\author{
Ji-Gang Zhang, Xue Zhang, Qin Li, Gao-Lin Liu \\ Department of Clinical Pharmacy, Shanghai General Hospital, Shanghai Jiao Tong University School of medicine, \\ China
}

Our previous study reported that rho-associated coiled-coil containing kinases (ROCKs) were involved in Vasculogenic mimicry (VM) formation in hepatocellular carcinoma (HCC). This study focused on whether the potential activator ras homolog family member $\mathrm{C}(\mathrm{RhoC})$ plays an essential role in VM via two isoforms of ROCK (ROCK1 and ROCK2). VM was presented in tumor tissues rather than non-cancerous tissues, which was positively correlated with clinical stage $(\mathrm{P}=$ 0.006). RhoC expression was up regulated in HCC tissues, especially the VM-positive (VM+) group, compared to noncancerous tissues $(\mathrm{P}<0.01)$, and patients with high expression of RhoC had shorter survival times $(\mathrm{P}<0.001)$. The knockdown of RhoC via the short hairpin RNA (shRNA) in SK-Hep-1 significantly decreased VM formation and cell motility. On contrary, cell motility and VM formation were remarkably enhanced when overexpressed RhoC in HepG2. Compared to ROCK1 shRNA, ROCK2 shRNA could largely affects VM formation, cell motility and the key VM factors and the epithelial-mesenchymal transition (EMT) markers in vitro and in vivo. Additionally, RhoC/ROCK2 promoted $\mathrm{VM}$ via ERK/MMP and FAK/paxillin signaling pathways. Our results show that RhoC/ROCK2 may have a major effect on VM in HCC and might be a potential therapeutic target for the treatment of HCC. 